\begin{tabular}{|c|c|}
\hline Title & Optical anisotropy and photoluminescence polarization in single InA IA s quantum dots \\
\hline Author(s) & Ohno, S.; A dachi, S.; Kaji, R.; Muto, S.; Sasakura, H. \\
\hline Citation & $\begin{array}{l}\text { A pplied Physics Letters, 98(16), } 161912 \\
\text { https://doi.org/10.1063/1.3583453 }\end{array}$ \\
\hline Issue Date & 2011-04 18 \\
\hline DOC URL & http:/hdl.handle.net/2115/45386 \\
\hline Rights & $\begin{array}{l}\text { Copy right } 2011 \text { A merican Institute of Physics. This article may be downloaded for personal use only. A ny other use } \\
\text { requires prior permission of the author and the A merican Institute of Physics. The following article appeared in A ppl. } \\
\text { Phys. Lett. } 98,161912 \text { and may be found at https://dx.doi.org } / 10.1063 / 1.3583453\end{array}$ \\
\hline Type & article \\
\hline File Information & APL98-16_161912.pdf \\
\hline
\end{tabular}

Instructions for use 


\title{
Optical anisotropy and photoluminescence polarization in single InAIAs quantum dots
}

\author{
S. Ohno, ${ }^{1}$ S. Adachi, ${ }^{1, a)}$ R. Kaji, ${ }^{1}$ S. Muto, ${ }^{1}$ and H. Sasakura ${ }^{2}$ \\ ${ }^{1}$ Department of Applied Physics, Hokkaido University, N13 W8, Kitaku, Sapporo 060-8628, Japan \\ ${ }^{2}$ Research Institute of Electronic Science, Hokkaido University, N21 W10, Kitaku, Sapporo 001-0021, Japan
}

(Received 11 March 2011; accepted 4 April 2011; published online 22 April 2011)

\begin{abstract}
We have investigated the optical anisotropy in individual self-assembled quantum dots. The linear polarization analysis of the positive trion photoluminescence reveals the effect of the strain-induced valence band mixing since the positive trion has the spin-paired holes and therefore exchange interaction has no influence. Meanwhile, the neutral exciton indicates the complex polarization states due to both the in-plain asymmetries of the dot shape and the strain distributions. The experimental and theoretical polarization analysis has been performed for tens of InAlAs quantum dots and the correlation between the important parameters was investigated. (C) 2011 American Institute of Physics. [doi:10.1063/1.3583453]
\end{abstract}

Studies on localized spins in semiconductor quantum dots (QDs) have been attracting considerable interest. This is because the discrete electronic levels involved in the optical transitions serve the fascinating applications in which QDs are used as emitters of single, indistinguishable, and entangled photons. ${ }^{1}$ For these applications, it is crucial to study the polarization of the emitted photons associated with exciton annihilation. For ideal QDs as artificial atoms, the relevant eigenstates are bright excitons with the angular momentum of \pm 1 , and the circularly polarized photons $\left(\sigma_{+}\right.$or $\left.\sigma_{-}\right)$are to be absorbed (emitted) to (from) the eigenstates. However, actual QDs have the anisotropic distributions of shape and strain, and as a result, the confinement potential symmetry is reduced from $D_{2 d}$ to $C_{2 v}$ or lower. It is well known that the shape anisotropy induces the change in the emission polarization as well as the level splitting as an exciton fine structure. ${ }^{2-5}$ Also, QDs formed by self-assembly in the Stransky-Krastanov (SK) growth mode is believed to have a large strain originating from the QD formation process, and the strain with the anisotropic distribution more or less remains inside a QD even after QD formation is complete. Consequently, the emission polarization is affected by the anisotropic exchange interaction (AEI) and the straininduced valence-band mixing (SI-VBM). The former originates from the QD shape asymmetry and the latter comes mainly from the in-plane anisotropic relaxation of strains. Accordingly, the polarization of the QD emissions is one of valuable probes for the origin of the QD symmetry lowering. Since the strain anisotropy may be different largely from QD to $\mathrm{QD}$, the investigation of the polarization is necessary for the individual QDs.

In this work, we investigated the polarization of the photoluminescence (PL) in single InAlAs QDs in order to probe the interactions that reduce the QD potential symmetry. By comparing the positive trion PL with the neutral exciton PL in the same single QD, the intrinsic parameters for AEI and SI-VBM were deduced. Since III-V semiconductor QDs generally have a smaller strain anisotropy than that in II-VI $\mathrm{QDs},{ }^{6,7}$ the precise estimation of the composite of AEI and SI-VBM in III-V semiconductor QDs have not been reported

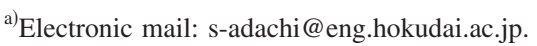

so far. The simple method presented here gives a clear perspective on the polarization states in the self-assembled QDs.

Self-assembled $\operatorname{In}_{0.75} \mathrm{Al}_{0.25} \mathrm{As}$ QDs were used for the experiments. The QDs were grown on an $\mathrm{Al}_{0.3} \mathrm{Ga}_{0.7} \mathrm{As}$ layer on an undoped (100) GaAs substrate in the SK growth mode by molecular beam epitaxy. From the atomic force microscopy (AFM) measurements, the average QD diameter (height) of $\sim 20(\sim 4) \mathrm{nm}$ and the weak in-plane asymmetry were found. The micro-PL measurements were performed at $6 \mathrm{~K}$ under a zero magnetic field. A cw-Ti:sapphire laser was tuned to $\sim 728 \mathrm{~nm}$, which gives the transition energy at the wetting layer. The unpolarized excitation was employed in order to ignore the dynamical process depending on the initial polarization and to reflect directly the ground state basis to the PL. The high-quality unpolarized light was made as a temporal averaging nonpolarization by an electro-optic modulator with the modulation period of $0.2 \mathrm{~ms}$ that was much shorter than the accumulation time (1 s.) of a Si-charge-coupled device detector. The degree of linear polarization of the PL was analyzed by using a set of a rotating half-wave plate and a fixed polarizer in front of a triple grating spectrometer. The spectral resolution that determines the emission peak energies was $\leq 5 \mu \mathrm{eV}$ by the spectral fitting. The details of various methods for the spectral peak assignment are seen in Refs. 8-10.

Here, we consider briefly how SI-VBM and AEI change the polarization of the QD emissions. First, we analyze the $\mathrm{PL}$ of a positive trion $\left(\mathrm{X}^{+}\right)$. The ground state of $\mathrm{X}^{+}$has two holes in a spin singlet and one electron, and has a halfinteger total spin. Therefore, $\mathrm{X}^{+}$does not split irrespective of the QD symmetry under no magnetic field according to Kramers theorem. However, inhomogeneous strain distribution gives rise to the anisotropic heavy hole (hh)-light hole (lh) mixing and changes the $\mathrm{X}^{+} \mathrm{PL}$ from circular to elliptical polarization. Such a SI-VBM can be treated by the Bir-Pikus Hamiltonian $(8 \times 8)$, where the ratio of the strain strength $\rho_{\mathrm{s}}$ to the hh-lh splitting energy $\Delta_{l h}$, i.e., $\rho_{\mathrm{s}} / \Delta_{l h}$, determines the degree of mixing., ${ }^{4,8}$ As a result, mainly hh and mainly $\mathrm{lh}$ exciton states, which are doubly degenerate, respectively, are generated. Assuming a small $\rho_{\mathrm{s}} / \Delta_{l h}$, the mainly hh excitons of our interest here can be represented as follows: 
(a)

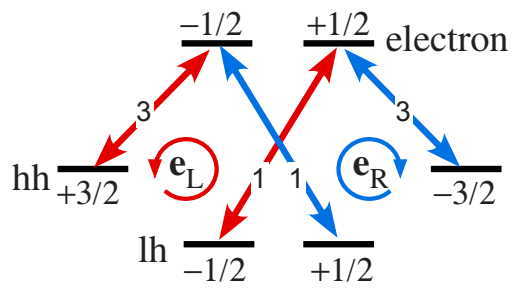

(b)

(c)

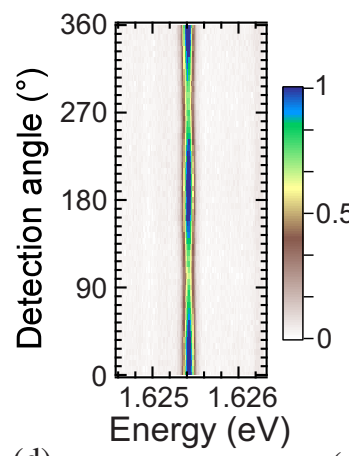

(d)

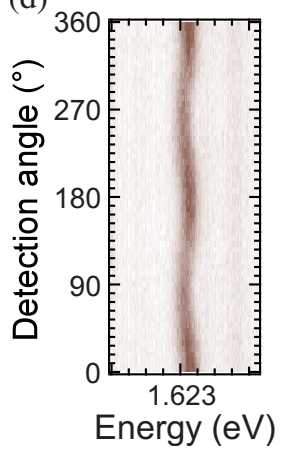

(e)
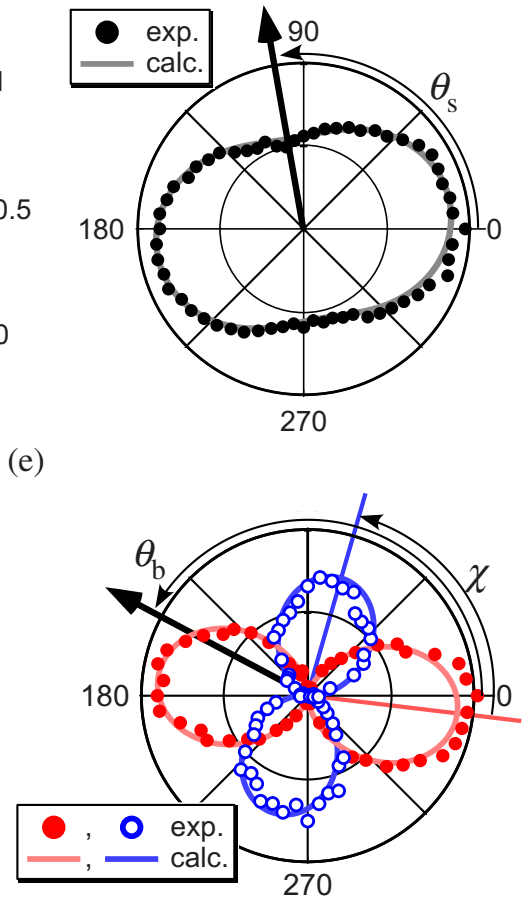

FIG. 1. (Color online) (a) Electron, hh, and lh levels and the optical allowed transitions. The transition probability ratios are also depicted. (b) $2 \mathrm{D}$ plot of the $\mathrm{X}^{+}$PL spectrum. (c) Polar plot of $\mathrm{X}^{+} \mathrm{PL}$ intensity. $\theta_{\mathrm{s}}$ is the strain orientation. (d) 2D plot of the $\mathrm{X}^{0} \mathrm{PL}$ spectrum. The energy scale is the same as that in (b). (e) Polar plots of the PL intensities of the $\mathrm{X}^{0}$ doublet. $\theta_{\mathrm{b}}$ is the dot shape anisotropy orientation. The angle $\chi$ is the angle difference between the polarization axes of the $\mathrm{X}^{0}$ doublet. All angles are measured in the same laboratory frame.

$$
\left|\varphi_{1(2)}^{\mathrm{e}-\mathrm{hh}}\right\rangle \sim\left| \pm \frac{3}{2}, \mp \frac{1}{2}\right\rangle-\frac{\rho_{\mathrm{s}}}{\Delta_{l h}} \mathrm{e}^{ \pm i 2 \theta_{s}}\left|\mp \frac{1}{2}, \mp \frac{1}{2}\right\rangle,
$$

where $\theta_{\mathrm{s}}$ is a half of the coupling phase that gives the principal axis of the strain distribution, $\left|J_{z}, S_{z}\right\rangle=| \pm 3 / 2, \mp 1 / 2\rangle$ and $|\mp 1 / 2, \mp 1 / 2\rangle$ are $h$ and $\mathrm{lh}$ excitons, respectively. In the absorption or emission of $\sigma_{+}\left(\sigma_{-}\right)$photons, the hh-lh mixing couples the electron states with $S_{z}=-1 / 2(+1 / 2)$ not only to the hh states with $J_{z}=+3 / 2(-3 / 2)$ but also to the $\mathrm{lh}$ states with $J_{z}=-1 / 2(+1 / 2)$ as shown in Fig. 1(a). From Eq. (1) and Fig. 1(a), the PL from the states can be written as

$$
\left|\boldsymbol{e}_{L(R)}-\frac{1}{\sqrt{3}} \frac{\rho_{\mathrm{s}} A}{\Delta_{l h}} \mathrm{e}^{ \pm i 2 \theta_{s}} \boldsymbol{e}_{R(L)}\right|^{2},
$$

where $\boldsymbol{e}_{L(R)}$ is a left (right) helicity basis of $\sigma_{+}\left(\sigma_{-}\right)$light and a coefficient $A$ is the ratio of the overlap integrals of electron and hh (lh) wave-functions $\left(\phi_{\mathrm{e}}\right.$ and $\left.\phi_{\mathrm{hh}(\mathrm{lh})}\right): A$ $=\int \phi_{\mathrm{e}}(\boldsymbol{r}) \phi_{\mathrm{lh}}(\boldsymbol{r}) d \boldsymbol{r} / \int \phi_{\mathrm{e}}(\boldsymbol{r}) \phi_{\mathrm{hh}}(\boldsymbol{r}) d \boldsymbol{r}$. The details of the calculations are seen in Refs. 4, 6, and 8. Though VBM also occurs without strain via the off-diagonal coupling in the LuttingerKohn Hamiltonian, the observed results shown later cannot be reproduced without assuming an largely elongated potential. ${ }^{11}$ Such a large shape anisotropy was not consistent with the AFM observation in InAlAs QDs.

Figure 1(b) is a two-dimensional (2D) plot of $\mathrm{X}^{+} \mathrm{PL}$ spectrum as a function of twice the angle of a half-wave plate rotation. A clear intensity modulation is seen depending on the detection angle and $\mathrm{X}^{+} \mathrm{PL}$ is found not to be perfectly circularly polarized. This change to the elliptical polarization is due to the VBM effect represented by Eq. (1). The polar plot of the $\mathrm{X}^{+} \mathrm{PL}$ intensity and the theoretical fitting [Fig. 1(c)] indicate the DLP $25 \%$ and the strain orientation $\theta_{\mathrm{s}}$ $\sim 100^{\circ} \pm 3^{\circ}$ in the laboratory frame. From the fitting, the parameter $\rho_{\mathrm{s}} A / \Delta_{\mathrm{lh}} \sim 0.22$ was also obtained. The value is smaller than CdTe/ZnTe QDs $(\sim 0.75)$ (Ref. 6) and CdSe/ ZnSe QDs $(\sim 0.3) .^{7}$ The deduced $\rho_{\mathrm{s}} A / \Delta_{\mathrm{lh}}$ of the QD shown in Fig. 1 is the largest value among the observed QDs that are summarized later (Fig. 3). Also the $\mathrm{X}^{+}$binding energy $E_{\mathrm{b}}\left(\mathrm{X}^{+}\right)$should be referred, which is used later as a measure of a hh mass affected by the hh-lh mixing. As shown in Figs. $1(\mathrm{~b})$ and $1(\mathrm{~d}), E_{\mathrm{b}}\left(\mathrm{X}^{+}\right)=-2.34 \mathrm{meV}$, where the negative sign means that the $\mathrm{X}^{+}$PL appears in the high energy side of the $\mathrm{X}^{0} \mathrm{PL}$.

The PL polarization of a neutral exciton $\mathrm{X}^{0}$ is affected by both AEI and SI-VBM, and a complex change in the polarization is expected depending on the magnitudes and orientations of both effects. Figures 1(d) and 1(e) show a 2D plot of $X^{0}$ PL spectrum and a polar plot of the PL intensities, respectively. The AEI splits the $\mathrm{X}^{0}$ states to the radiative doublet separated by the energy $\delta_{\mathrm{b}}$ and mixes the original eigenstates $| \pm 1\rangle$ to $\left(|+1\rangle \pm e^{i 2 \theta_{\mathrm{b}}}|-1\rangle\right) / \sqrt{2}$. $^{4,5}$ The $\mathrm{X}^{0}$ PL polarization as shown in Fig. 1(e), therefore, is quite different from that of $\mathrm{X}^{+}$[Fig. 1(c)]. Here, $\theta_{\mathrm{b}}$ describes a half of the coupling phase and determines the orientation of a QD shape asymmetry. When only the AEI affects $\mathrm{X}^{0}$ states (no SIVBM), the polarization of the doublet is orthogonal to each other, and the PL intensities of the doublet are equivalent if Boltzmann distribution at the thermal equilibrium can be ignored. From the definition, $\theta_{\mathrm{b}}$ coincides with the polarization axis of the high energy level of the doublet in the case of $\delta_{\mathrm{b}}>0$. The anisotropic splitting energy $\delta_{\mathrm{b}}$ originates from QD elongation and/or interface optical anisotropy and varies from 5 to $\sim 200 \mu \mathrm{eV}$ in InAlAs QDs. ${ }^{12}$

When the effect of the SI-VBM is combined with the $\mathrm{AEI}$, the $\mathrm{X}^{0} \mathrm{PL}$ properties are found to change in the following points from the experiments and the calculations:

(1) PL intensities of the doublet become uneven.

(2) Polarizations of the doublet deviate from the orthogonal polarization.

(3) Polarization axis of the high energy level deviates from the shape anisotropy orientation $\theta_{\mathrm{b}}$.

(4) The observed splitting energy deviates from the value of $\delta_{\mathrm{b}}$.

While the $\mathrm{X}^{0}$ doublet in Fig. 1(e) was linearly polarized, the angle between the polarization axes $\chi$ was $81^{\circ} \pm 3^{\circ}$ and had the different PL intensities. The angle $\theta_{\mathrm{b}}\left(=152^{\circ}\right)$ stirs from the polarization axis of the high energy level. In addition, the splitting energy observed directly from Fig. 1(d) $(70 \mu \mathrm{eV})$ was different from the $\delta_{\mathrm{b}}(43 \mu \mathrm{eV})$ obtained by the best fitting, which is indicated by the solid curves in Fig. $1(\mathrm{e})$. 

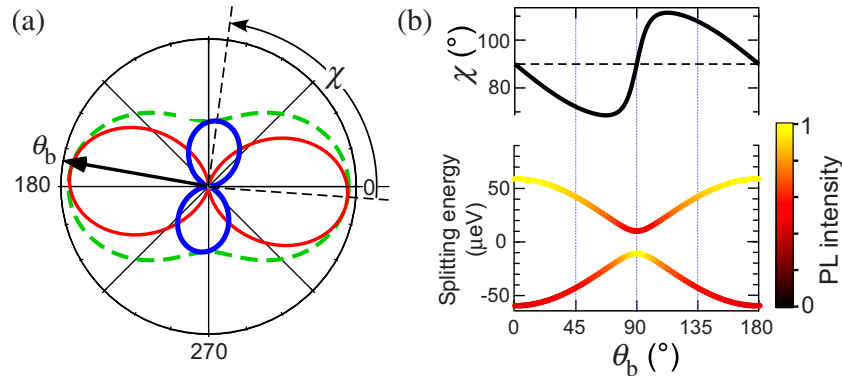

FIG. 2. (Color online) (a) An example of the $\mathrm{X}^{0}$ polarization states affected by both $\operatorname{VBM}\left(\theta_{\mathrm{s}}=90^{\circ}\right)$ and $\operatorname{AEI}\left(\theta_{\mathrm{b}}=170^{\circ}\right)$ with the medium strain strength $\left(\rho_{\mathrm{s}} A / \Delta_{\mathrm{lh}}=0.36\right)$. (b) In the case of the fixed $\theta_{\mathrm{s}}=90^{\circ}$, the calculated splitting energy and PL intensity (see the color table) of the $\mathrm{X}^{0}$ doublet are plotted as a function of $\theta_{\mathrm{b}}$. The following main parameters are used; $\rho_{\mathrm{s}} A / \Delta_{l h}=0.36$, $\delta_{\mathrm{b}}=50 \mu \mathrm{eV}, \delta_{0}=400 \mu \mathrm{eV}, \delta_{\mathrm{d}}=5 \mu \mathrm{eV}, \theta_{\mathrm{s}}=90^{\circ} .{ }^{13}$

These features due to the SI-VBM become clearer with increasing $\rho_{\mathrm{s}} A / \Delta_{\mathrm{lh}}$. Figure 2 shows a typical example of the composite effect of AEI and SI-VBM with a medium strain strength $\left(\rho_{\mathrm{s}} A / \Delta_{\mathrm{lh}}=0.36\right)$. In Fig. 2(a), a thick solid (blue) and a thin solid (red) lines indicate the PL intensities of the low and high energy states in the $\mathrm{X}^{0}$ doublet, respectively, and a broken line (green) shows a total PL intensity of $\mathrm{X}^{0}$. Figure and other calculated results (not shown here) indicate that the total PL polarization is decided by the SI-VBM like a broken curve in Fig. 2, and the respective PL polarization of the $\mathrm{X}^{0}$ doublet moves within the frame depending on the shape anisotropy orientation $\theta_{\mathrm{b}}$ and the AEI strength $\delta_{\mathrm{b}}$. As shown in Fig. 2(b), the effect of the SI-VBM changes drastically depending on the relative angle between $\theta_{\mathrm{s}}$ and $\theta_{\mathrm{b}}$ even in the case that the remained parameters are fixed. Remarkable changes appear in the PL intensities, the polarization angle, and the splitting energy of the $\mathrm{X}^{0}$ doublet. At $\theta_{\mathrm{b}}$ $=\theta_{\mathrm{s}} \pm 90^{\circ} n$, the splitting energy of the doublet becomes minimum in $n=$ even integers and maximum in $n=$ odd integers. Also, the PL intensity difference changes to maximum around $\theta_{\mathrm{b}}=\theta_{\mathrm{s}} \pm 90^{\circ} n$ ( $n=$ all integers). In particular, the high energy PL peak becomes large at $\theta_{\mathrm{b}} \sim \theta_{\mathrm{s}} \pm 90^{\circ} n$ ( $n=$ odd integers) regardless of the largest splitting energy. Furthermore, the polarization axes of the $\mathrm{X}^{0}$ doublet are not orthogonal other than $\theta_{\mathrm{b}}=\theta_{\mathrm{s}} \pm 90^{\circ} n$ ( $n=$ all integers). These results mean that the degree of $\mathrm{lh}-\mathrm{hh}$ mixing depends strongly on the orientations of the shape and strain anisotropy distributions $\left(\theta_{\mathrm{b}}\right.$ and $\left.\theta_{\mathrm{s}}\right)$. The aforementioned features of the combined effect are indicated quite clearly in this figure and is found even in the data in Fig. 1 with following the small $\rho_{\mathrm{s}} A / \Delta_{\mathrm{lh}}$.

The next interest is a correlation among the important parameters of many QDs. For the purpose, the thirteen InAlAs QDs in the same sample were analyzed in the same manner as Fig. 1. The $\mathrm{X}^{0}$ and $\mathrm{X}^{+}$PL peaks were observed in the range of 1.610-1.650 eV. Figure 3(a) shows the orientations of $\theta_{\mathrm{b}}$ and $\theta_{\mathrm{s}}$. In the investigated QDs, both angles have the dense distributions around $90^{\circ}$ and $170^{\circ}$ with the similar dispersion of $\sim 40^{\circ}$, although there exists no definite correlation between $\theta_{\mathrm{b}}$ and $\theta_{\mathrm{s}}$ of individual QDs. Meanwhile the moderate correlation is found in the $\mathrm{X}^{+}$binding energy and $\theta_{\mathrm{s}}$ against $\rho_{\mathrm{s}} A / \Delta_{\mathrm{lh}}$ as shown in Fig. 3(b). The qualitative explanation for a part of this result can be given as follows.
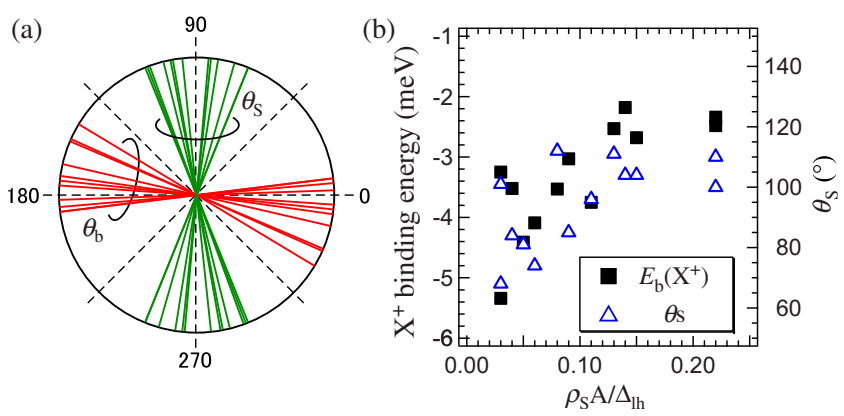

FIG. 3. (Color online) (a) The orientations of $\theta_{\mathrm{s}}$ and $\theta_{\mathrm{b}}$ in the observed QDs are indicated by the solid lines, respectively. (b) The binding energy of $\mathrm{X}^{+}$ and $\theta_{\mathrm{s}}$ vs $\rho_{\mathrm{s}} A / \Delta_{\mathrm{lh}}$.

The $\mathrm{X}^{+}$binding energy reflects the magnitude of the repulsion acts between an exciton and an additional hole of $\mathrm{X}^{+}$in a QD, where a hole wave-function is confined more closely than an electron due to its heavy mass. Since the $\mathrm{lh}$ mixing to hh makes the mainly hh mass lighter and the confinement of the mainly hh becomes weak with increasing $\rho_{\mathrm{s}} A / \Delta_{\mathrm{lh}}$, the repulsive force reduces and therefore the absolute value of the binding energy decreases. The correlation between $\theta_{\mathrm{s}}$ and $\rho_{\mathrm{s}} A / \Delta_{\mathrm{lh}}$ is not explained yet at this stage. The details including the quantitative explanation will be reported elsewhere.

In summary, in terms of the shape anisotropy that induces AEI and the strain anisotropy that induces VBM, we could explain well the observed polarization states of neutral excitons and positive trions in individual InAlAs QDs. The composite effect of AEI and SI-VBM in the neutral exciton PL was fully investigated and the method gives a clear perspective on the polarization states in the self-assembled QDs.

We acknowledge Y. Toda for fruitful discussions. This work was supported in part by the Grant-in-Aid for Scientific Research (Grant No. 22310063) from the Ministry of Education, Culture, Sports, Science, and Technology, Japan.

${ }^{1}$ O. Benson, C. Santori, M. Pelton, and Y. Yamamoto, Phys. Rev. Lett. 84, 2513 (2000).

${ }^{2}$ D. Gammon, E. S. Snow, B. V. Shanabrook, D. S. Katzer, and D. Park, Phys. Rev. Lett. 76, 3005 (1996).

${ }^{3}$ M. Bayer, G. Ortner, O. Stern, A. Kuther, A. A. Gorbunov, A. Forchel, P. Hawrylak, S. Fafard, K. Hinzer, T. L. Reinecke, S. N. Walck, J. P. Reithmaier, F. Klopf, and F. Schäfer, Phys. Rev. B 65, 195315 (2002).

${ }^{4}$ E. L. Ivchenko, Optical Spectroscopy of Semiconductor Nanostructures (Alpha Science International, Harrow, UK, 2005).

${ }^{5}$ Spin Physics in Semiconductors, edited by M. I. Dyakonov (Springer, Berlin, 2008), Chap. 4.

${ }^{6}$ Y. Léger, L. Besombes, L. Maingault, and H. Mariette, Phys. Rev. B 76, 045331 (2007).

${ }^{7}$ A. V. Koudinov, I. A. Akimov, Y. G. Kusrayev, and F. Henneberger, Phys. Rev. B 70, 241305(R) (2004).

${ }^{8}$ R. Kaji, Doctoral thesis, Hokkaido University, 2011.

${ }^{9}$ H. Kumano, S. Kimura, M. Endo, H. Sasakura, S. Adachi, S. Muto, and I. Suemune, J. Nanoelectron. Optoelectron. 1, 39 (2006).

${ }^{10}$ S. Adachi, N. Yatsu, R. Kaji, S. Muto, and H. Sasakura, Appl. Phys. Lett. 91, 161910 (2007)

${ }^{11}$ T. Tanaka, J. Singh, Y. Arakawa, and P. Bhattacharya, Appl. Phys. Lett. 62, 756 (1993)

${ }^{12}$ R. Kaji, S. Adachi, S. Shindo, and S. Muto, Phys. Rev. B 80, 235334 (2009).

${ }^{13}$ The parameters except for $\rho_{\mathrm{s}} A / \Delta_{\mathrm{lh}}$ are observed ones for hh excitons in InAlAs QDs. Also, the parameters for $\mathrm{lh}$ excitons $\delta_{\mathrm{b}}^{\mathrm{h}}=250 \mu \mathrm{eV}, \delta_{0}^{\mathrm{h}}$ $=800 \mu \mathrm{eV}, \delta_{\mathrm{d}}^{\mathrm{h}}=100 \mu \mathrm{eV}$ were used in the calculation. 